\title{
A Gravidez não planejada de mulheres atendidas no pré-natal das Unidades Básicas de Saúde
}

\author{
Clícia Dias da Silva Rodrigues ${ }^{1}$; Arianna Oliveira Santana Lopes ${ }^{2}$
}

\begin{abstract}
Resumo: A gravidez não planejada é toda a gestação que não foi programada, ou que foi causada por uma necessidade insatisfeita de contracepção. Os desdobramentos de sua ocorrência, atualmente são considerados grandes demandas para a saúde pública, principalmente entre as mulheres mais jovens e nas famílias em vulnerabilidade social (FINOTTI, 2015). Tendo em vista estes aspectos, o objetivo deste estudo foi averiguar os aspectos relacionados a gravidez não planejada em mulheres atendidas no pré-natal, apresentando a importância da atuação do enfermeiro no tocante a prevenção, orientação e controle, em unidades de saúde de cidades da região sudoeste da Bahia. Trata-se de um estudo exploratório e descritivo com corte transversal analítico, cuja abordagem é quantitativa, onde os dados serão oriundos da aplicação de um questionário estruturado, dividido em 20 questões, que versam sobre as características socioeconômicas e demográficas, e da história contraceptiva e gestacional da mulher. A pesquisa transcorreu entre os meses de julho e setembro de 2016, frente a uma amostra de 100 mulheres gravidas. A análise dos dados do estudo foi realizada de forma descritiva, utilizando a tabulação de dados, com média, desvio padrão, porcentagem e frequência através do programa Excel 2013 que em seguida foram submetidos ao software SPSS 23.0. Para tanto, um cálculo amostral foi realizado baseando-se no erro amostral de $5 \%$ e no nível de confiança de $90 \%$, dentre as mulheres entrevistadas. O resultado desse estudo evidencia que as mulheres que apresentam gravidez indesejada, em sua grande maioria, utilizavam métodos contraceptivos de forma equivocada, fato que causou a gravidez. Além disso, aponta-se para uma precária participação das mulheres no planejamento familiar e também na visitação das equipes de saúde da família. Esse estudo conclui assim, que essa utilização errônea dos métodos contraceptivos estão relacionados diretamente com a falta de informação e desconhecimento sobre o método, devido a aspectos socioeconômicos, bem como a ineficiência do sistema de saúde e suas instituições, que não conseguem fornecer a instrução necessária a população feminina sobre a prevenção da gravidez não planejada.
\end{abstract}

Palavras-Chave: Gravidez não Planejada; Unidades básicas de Saúde, Planejamento Familiar; Enfermagem.

\section{The Unplanned Pregnancy of Women Served at the Prenatal Care of the Basic Health Units}

Unwanted pregnancy is any pregnancy that was not scheduled, or was caused by an unmet need for contraception. The consequences of its occurrence, are currently considered high demands for public health, especially among younger women and families in social vulnerability (FINOTTI, 2015). Considering these aspects, the aim of this study was to investigate aspects related to unplanned pregnancies in women attending prenatal, showing the importance of the work of nurses regarding the prevention, guidance and control, in cities of health units southwest region of Bahia. This is an exploratory and descriptive study with analytical cross-section, whose approach is quantitative, where the data will come from the application of a structured questionnaire, divided into 20 questions, which deal with the socioeconomic and demographic characteristics and contraceptive history and gestational woman. The survey went between the months of July and September 2016, compared to a sample of 100 pregnant women. The analysis of the study data were descriptively, using the tab data, mean, standard deviation, percentage and frequency through the Excel 2013 program which were then submitted to the SPSS 23.0 software. Thus, a sample size calculation was performed based on the sampling error of 5\% and a confidence level of $90 \%$ among the women interviewed. The result of this study shows that women who have unwanted pregnancies, mostly, used

\footnotetext{
${ }^{1}$ Bacharelanda do curso de Enfermagem da Faculdade Independente do Nordeste - FAINOR. E-mail: clicia93@ @otmail.com;

${ }^{2}$ Mestre. Professora do Curso de Enfermagem da Faculdade Independente do Nordeste (FAINOR). ariannasantana@bol.com.br.
} 
Id on Line Revista Multidisciplinar e de Psicologia

Id on Line Multidisciplinary and Psycology Journal

contraceptive methods in error, a fact that caused the pregnancy. In addition, it is noted for poor participation of women in family planning and also the visitation of family health teams. This study concludes that this erroneous use of contraceptive methods are directly related to the lack of information and ignorance about the method, due to socio-economic aspects as well as the inefficiency of the health system and its institutions, which fail to provide the necessary education the female population on the prevention of unplanned pregnancy.

Key Words: unplanned pregnancy; Basic Units of Health, Family Planning; Nursing.

\section{Introdução}

A gravidez não planejada ou não pretendida pode ser conceituada como resultado direto de uma necessidade insatisfeita de contracepção, e devido a seus desdobramentos, atualmente se encontra entre os principais problemas de saúde pública no mundo contemporâneo, principalmente para as mulheres mais jovens e nas famílias em situação de vulnerabilidade social (FINOTTI, 2015).

Em termos conceituais, cabe salientar que muitos dos estudos realizados no Brasil não fazem distinção entre gestações "não planejada" e "não desejada", que são usados como sinônimos. Prietsch et al. (2011), em um estudo no Sul do Brasil, utilizaram o termo "não planejada", para se referir àquelas gestações que não foram programadas pela mulher ou pelo casal, e "inoportunas" como sendo aquelas que ocorreram em época diferente da desejada.

Nesse sentido, comprovando o grau de incidência dessa problemática, Finer e Zolna (2011), apontam que nos Estados Unidos, por exemplo, a gravidez não planejada representa metade de todas as gestações. Assim, mesmo reconhecendo essa problemática, que atinge diretamente a saúde da mulher e a instituição familiar, poucos são os estudos relacionados à demanda, existindo muita discussão sobre suas causas, fatores associados e como este tema deve ser abordado.

Sobre essa questão enumera-se aqui que os estudos sobre esse tema promoveram a mobilização da área da saúde, no tocante a elaboração e execução de ações que visavam à redução do número de gestações não planejadas. No Brasil nota-se a execução de campanhas, do Ministério da Saúde (MS), pelo planejamento familiar, com o objetivo de promoção à saúde da mulher, buscando um aumento na proporção de gestações desejadas, visto que o grande 
Id on Line Revista Multidisciplinar e de Psicologia

Id on Line Multidisciplinary and Psycology Journal

número de gravidezes não planejadas é um importante indicador da falha no controle do processo reprodutivo e consequentemente do acompanhamento das famílias (FINOTTI, 2015).

Como prova do grande problema que a gravidez não planejada é, Langer (2002 apud PRIESTCH et al, 2011), aponta que a cada ano, pelo menos 80 milhões de mulheres em todo o mundo experimentam a situação de ter uma gravidez não planejada, número que vem crescendo nas últimas décadas. Ainda apresentando dados sobre essa temática, Bayer (2014), enumera que 55\% das grávidas brasileiras não têm gestação planejada, como aponta o estudo "Nascer no Brasil", onde 76\% das entrevistadas, afirmam que uma gravidez não planejada atrapalharia os planos de uma vida futura. Quando se trata do público adolescente, a gravidez não planejada pode ser ainda mais devastadora. Segundo relatório "Situação da População Mundial 2013”, do Fundo de População das Nações Unidas (UNFPA), cerca de 20 mil meninas, com menos de 18 anos, dão a luz todos os dias nos países em desenvolvimento (BAYER, 2014).

Entre as principais medidas adotadas para enfrentamento do problema da gravidez não planejada, pode-se apontar o aumento do conhecimento em saúde reprodutiva, contracepção e gravidez não planejada; a melhoria do acesso a métodos contraceptivos, o desenvolvimento e avaliação criteriosa de programas locais para reduzir o numero de gestações não planejadas; a melhor organização dos serviços de planejamento familiar; e o entendimento dos determinantes e antecedentes da gravidez não planejada (INSTITUTE OF MEDICINE, 1995 apud BRITO, 2013). Como ferramenta fundamental de implantação dessas medidas tem-se o Programa de Planejamento Familiar do ministério da Saúde e o enfermeiro que desempenha de forma crucial atividades no planejamento familiar dessas mulheres.

Diante dessas concepções e entendendo que a problemática da gravidez não planejada influencia diretamente na saúde da mulher e na sua estrutura familiar, esse estudo se justificou pela necessidade de identificação e análise das principais causas desse fenômeno, para entendimento dessa demanda e apresentação de possíveis ações que possam minimizar o número expressivo de mulheres grávidas que buscam atendimento no pré-natal nas unidades básicas de saúde.

O estudo buscou ainda a identificação de características sociodemográficas das mulheres grávidas atendidas na consulta do pré-natal, para assim apresentar o perfil epidemiológico das gestantes e os principais fatores associados a essa demanda. 
Id on Line Revista Multidisciplinar e de Psicologia

Id on Line Multidisciplinary and Psycology Journal

Assim, diante da complexidade e grande incidência da gravidez não planejada, além da necessidade de conhecer alternativas e possibilidades para a atuação do enfermeiro frente a essa demanda elaborou-se o seguinte problema de pesquisa: Quais as principais causas e aspectos que caracterizam a grande incidência da gravidez não planejada em mulheres atendidas no prénatal das unidades de saúde de cidades da região sudoeste da Bahia?

Para responder tal indagação, os objetivos traçados para elaboração desse estudo, visaram averiguação dos aspectos relacionados a gravidez não planejada em mulheres atendidas no pré-natal, apresentando a importância da atuação do enfermeiro no tocante a prevenção, orientação e controle, em unidades de saúde de cidades da região sudoeste da Bahia. Especificamente, o objetivo dessa produção textual é o de identificar a incidência e a as principais causas da gravidez não planejada, verificar os possíveis desfechos que essa gravidez pode ou poderia ter a partir da sua descoberta, apresentar os principais métodos contraceptivos disponibilizados nas unidades básicas de saúde, bem como identificar e relacionar o conhecimento e uso correto dos métodos contraceptivos com a gravidez não planejada através do discurso das gestantes.

Em termos de relevância do estudo, aponta-se que a relevância social do mesmo esta relacionada com a questão da falta de planejamento da gravidez, que pode promover a vulnerabilidade do grupo das mulheres quanto aos agravos de saúde, dos quais podem-se citar a mortalidade materna, os abortos provocados e algumas patologias infantis, visto que a mulher que não planejou muitas vezes não dispõe de uma estrutura mínima para o acompanhamento da gravidez, para a chegada do bebê e para a recuperação pós-parto. Já a relevância acadêmica está vinculada ao crescimento da demanda da gravidez não planejada que influencia diretamente nas questões relativas a saúde da mulher e da criança, e ao planejamento familiar, área diretamente associada ao trabalho de enfermagem que podem estar atrelados a falhas e devem ser desenvolvidos com precisão nas unidades de saúde.

Portanto, uma melhor compreensão dos fatores associados e o conhecimento da realidade regional sobre a gravidez não planejada podem auxiliar na compreensão dessa demanda, e posteriormente na elaboração, implantação e execução de políticas públicas e ações na área da saúde, voltadas para a atuação do enfermeiro no planejamento reprodutivo, e consequentemente na prevenção e controle da gravidez através do uso de métodos contraceptivos. 
Id on Line Revista Multidisciplinar e de Psicologia

Id on Line Multidisciplinary and Psycology Journal

\section{Metodologia}

Trata-se de um estudo exploratório e descritivo com corte transversal analítico, cuja abordagem é quantitativa.

O grupo de mulheres que participaram da pesquisa foi selecionado em duas unidades de saúde, localizadas nos municípios de Itambé-Bahia e de Vitória da Conquista-Bahia. Vitória da Conquista é o terceiro maior município da Bahia, com pouco mais de 340 mil habitantes (IBGE, 2016), já Itambé em uma população de aproximadamente 23 mil habitantes (IBGE, 2016), ambos os municípios são situados na região sudoeste da Bahia. A pesquisa transcorreu entre os meses de julho e setembro de 2016.

Para população deste trabalho, selecionou-se todas as mulheres gestantes, que referiram gravidez não programada, ou não desejada, e que eram atendidas nas consultadas de pré-natal desenvolvidas nas unidades de saúde no período da realização da pesquisa, numa amostra não probabilística por conveniência.

Inicialmente foram identificadas todas as gestantes que realizavam pré-natal nas unidades básicas de saúde por meio de busca em prontuários, em seguida selecionou-se 100 mulheres das que referiram gravidez não planejada durante a consulta de pré-natal. Essas foram recrutadas e após verificação de obediência aos critérios de inclusão e exclusão participaram da aplicação do questionário.

Utilizou-se como critérios de inclusão dos participantes: todas as mulheres deviam estar grávidas, ser de qualquer idade e relatar durante a consulta de pré-natal gravidez não programada, ou não planejada, devendo ainda aceitar participar do estudo. Quanto aos critérios de exclusão determina-se que não participaram do processo de pesquisa as gestantes que possuíam algum déficit de compreensão e dificuldades de responderem o questionário.

Após a seleção de todas as mulheres que relataram gravidez indesejada no momento da consulta foi aplicado, para coleta de dados, um questionário estruturado, dividido em 20 questões. Nele constam perguntas sobre as características socioeconômicas e demográficas da mulher, história contraceptiva, dados da gravidez atual, apoio social e familiar, pré-natal dividido por seções:

- Seção 1 - Características socioeconômicas e demográficas da mulher

- Seção 2 - História Contraceptiva e Gestacional 
Id on Line Revista Multidisciplinar e de Psicologia

Id on Line Multidisciplinary and Psycology Journal

A análise dos dados do estudo realizou-se de forma descritiva, utilizando a tabulação de dados, com média, desvio padrão, porcentagem e frequência através do programa Excel 2013 que em seguida foram submetidos ao software SPSS 23.0. Para tanto, um cálculo amostral foi realizado baseando-se no erro amostral de $5 \%$ e no nível de confiança de $90 \%$, dentre as mulheres entrevistadas.

As participantes da pesquisa foram devidamente esclarecidas quanto aos objetivos do trabalho, ficando livres para participarem ou não. Aceitando, assinaram o Termo de Consentimento Livre e Esclarecido TCLE, sendo respeitados os princípios éticos que constam na resolução 466/12 do Conselho Nacional de Saúde. A desistência ou não participação não implicou em prejuízo ao indivíduo na instituição.

O estudo foi submetido ao Núcleo de educação permanente e Comitê de Ética em pesquisa da Faculdade Independente do Nordeste (CEP/FAINOR) para autorização da pesquisa e aplicação do questionário.

\section{Resultados e Discussão}

Foram entrevistadas 100 (cem) mulheres, todas grávidas e que relataram para as enfermeiras responsáveis pelas unidades de saúde da família, durante o acompanhamento do pré-natal, ter uma gravidez indesejada, não programada, ou não planejada.

Nessa entrevista, na analise de dados socioeconômicos verificou-se que uma prevalência de grávidas, com idade entre os 20 e 30 anos, com escolaridade média de Ensino Médio Incompleto (27\%). Aponta-se ainda que a maioria das mulheres participantes do estudo possuem companheiro/marido (61\%), moram em domicilio próprio $(54 \%)$, num domicilio com média de 3 à 4 moradores (46\%), com religiões predominantemente católica (52\%) ou protestante $(45 \%)$. Como dados preocupantes observa-se que $46 \%$ da população entrevistada vivem com renda até 1 salário mínimo, que ainda se perpetua o grande número de mulheres grávidas com menos de 18 anos (21\% da amostra), e que a escolaridade é muito baixa entre as mulheres que apresentam a gravidez indesejada, onde $23 \%$ ainda não completaram sequer o Ensino Fundamental. Demais informações podem ser observadas na Tabela 1. 
Tabela 1. Características das variáveis socioeconômicas do estudo. Vitória da Conquista e Itambé, Bahia, Brasil, 2016.

\begin{tabular}{|c|c|c|}
\hline Variáveis & $\mathrm{N}$ & $\%$ \\
\hline \multicolumn{3}{|l|}{ Grupo Etário } \\
\hline menos de 18 anos & 21 & $21 \%$ \\
\hline entre 18-21 anos & 20 & $20 \%$ \\
\hline entre $22-25$ anos & 24 & $24 \%$ \\
\hline entre $26-29$ anos & 17 & $17 \%$ \\
\hline Acima de 30 anos & 18 & $18 \%$ \\
\hline \multicolumn{3}{|l|}{ Escolaridade } \\
\hline Fundamental Incompleto & 23 & $23 \%$ \\
\hline Fundamental Completo & 20 & $20 \%$ \\
\hline Médio Incompleto & 27 & $27 \%$ \\
\hline Médio Completo & 21 & $21 \%$ \\
\hline Superior Incompleto & 07 & $07 \%$ \\
\hline Superior Completo & 05 & $05 \%$ \\
\hline \multicolumn{3}{|l|}{ Estado Civil } \\
\hline Sem Marido/Companheiro & 39 & $39 \%$ \\
\hline Com Marido/Companheiro & 61 & $61 \%$ \\
\hline Tipo de Domicílio & 54 & $54 \%$ \\
\hline Próprio & 37 & $37 \%$ \\
\hline $\begin{array}{l}\text { Alugado } \\
\text { Cedido }\end{array}$ & 09 & $09 \%$ \\
\hline \multicolumn{3}{|l|}{ Moradores do Domicílio } \\
\hline $1-2$ pessoas & 20 & $20 \%$ \\
\hline 3-4 pessoas & 46 & $46 \%$ \\
\hline 5 ou mais pessoas & 34 & $34 \%$ \\
\hline \multicolumn{3}{|l|}{ Renda } \\
\hline Até 1 salário mínimo & $\begin{array}{l}40 \\
38\end{array}$ & $38 \%$ \\
\hline entre 2 e 3 salários mínimos & 14 & $14 \%$ \\
\hline $\begin{array}{l}\text { entre } 4 \text { e } 5 \text { salários mínimos } \\
\text { acima de } 5 \text { salário mínimo }\end{array}$ & 02 & $02 \%$ \\
\hline \multicolumn{3}{|l|}{ Religião } \\
\hline Não tem religião & 01 & $01 \%$ \\
\hline Católica & 52 & $52 \%$ \\
\hline Protestante & 45 & $45 \%$ \\
\hline Espírita & 01 & $01 \%$ \\
\hline Ubanda / Candomblé & 01 & $01 \%$ \\
\hline TOTAL & 100 & $100 \%$ \\
\hline
\end{tabular}

O perfil socioeconômico e reprodutivo das mulheres desse estudo envolveu uma amostra de mulheres jovens na primeira gravidez até mulheres com mais de 30 anos de idade, com história de gravidezes anteriores, solteiras e aquelas que moravam com parceiro, com renda financeira variável, e escolaridades diversas. Essa diversidade de condições sociais, 
Id on Line Revista Multidisciplinar e de Psicologia

Id on Line Multidisciplinary and Psycology Journal

relacionamentos pessoais, e de histórias reprodutivas torna-se relevante quando, pensa-se em mensurar o planejamento da gravidez, pois a idade, renda, escolaridade e o número de filhos estão positivamente associados à indesejabilidade da gravidez, como ressalta Berquó et al (2009).

Outros autores que falam sobre essas relações entre os dados socioeconômicos e reprodutivos são Evangelista, Barbieri e Silva (2015), que verificaram em seu estudo, que entre as mulheres em idade fértil, a maioria se encontrou em união estável. Essa situação, também é evidenciada nessa pesquisa, pois as mulheres que vivem a experiência da gravidez, em sua maioria, apresentam essa condição, ou seja, a ocorrência da gravidez não planejada e estado civil das mulheres tem relação direta, seja entre o grupo de mulheres com parceiro fixo, como àquelas sem parceiro fixo.

Sardinha et al (2014), em seu estudo sobre gravidezes indesejadas, apontam metade dessas gestações surgiram de um relacionamento não estável, demonstrando a visível ausência de planejamento familiar, que no futuro pode resultar em diversas demandas geradoras de vulnerabilidade e conflitos familiares.

Outro dado importante e que também encontrou similaridade em pesquisas se refere à relação em número de moradores do domicilio e a gravidez. Nesse sentido, o estudo foi similar ao de Prietsch et al (2011), que observou-se que quanto maior o número de moradores no domicílio, mais frequente foi a gravidez indesejada.

As informações contidas na Tabela 2, referem-se a situação da gravidez atual e do histórico de gravidezes da puérpera. Nota-se que a maioria da amostra (61\%) já teve outras gravidezes e que mais da metade das mulheres entrevistadas (54\%) apontam que sua gravidez atual além de não planejada, aconteceu sem o desejo, espera ou preparação da mulher e sua família. Além disso, esse estudo evidencia que a maior parte das mulheres (55\%) enumeram a falha do método contraceptivo como motivo de sua atual gravidez.

Tabela 2. Situação histórica e da atual gravidez apresentado pelas participantes do estudo. Vitória da Conquista e Itambé, Bahia, Brasil, 2016.

\begin{tabular}{lll}
\hline Variáveis & $\mathrm{N}$ & $\%$ \\
\hline Vezes que Engravidou & & \\
$\quad$ É a primeira gravidez & 39 & $39 \%$ \\
Já teve outras gravidezes & 61 & $61 \%$
\end{tabular}


Id on Line Revista Multidisciplinar e de Psicologia

Id on Line Multidisciplinary and Psycology Journal

\section{Gravidez Atual}

Não foi planejada, mas estava pensando

no assunto

Não foi planejada, não estava querendo

e nem estava preparado

\section{Motivo da gravidez}

Falta de uso de métodos contraceptivo

Falha no método contraceptivo utilizado

17

65

Não utilizou nenhum método contraceptivo

Percebe-se com os dados dessa tabela que as mulheres que apresentam gravidezes indesejadas ou não planejadas, em sua maioria já são mães. Essa mesma relação foi diagnostica por Prietsch et al (2011), onde relata-se que as mulheres com mais filhos têm mais gravidez indesejada, tal qual como evidenciado nessa pesquisa. No estudo de Nery et al (2015), apontase que a reincidência de gravidez não planejada está diretamente ligada as mulheres que não estudavam e apresentavam total dependência financeira, sendo que esses fatos tem-se mostrado como principal variável, pois quanto maior for o grau de instrução, maior será sua possibilidade de negociação do método contraceptivo e seu uso correto, tanto na primeira relação sexual como nas seguintes.

O fato de já ter vivenciado um período gestacional pode estar atrelado a sensação de confiança e uso indevido ou não uso do métodos.

Quanto a falta de planejamento da gravidez, o trabalho mostra que as mulheres não estavam preparadas para tal acontecimento, sendo que a maioria delas estava usando algum método contraceptivo e que o mesmo não foi utilizado corretamente.

Sardinha et al (2014), destaca em sua pesquisa uma evidenciação semelhante, pois enumera que embora toda a sua amostra (100\%), relatam que acham importante a utilização dos métodos contraceptivos para evitar uma gravidez, observou-se $61,7 \%$ dessas mulheres engravidaram por "descuido", que também pode ser compreendido como má utilização dos métodos contraceptivos ou mesmo a não utilização dos mesmos. Em outros estudos semelhantes, como os de Tachibana, Santos e Duarte (2006 apud SANCHES, 2013), confirmam-se esses resultados, evidenciando que parcelas significativas das mulheres engravidam por descuidos relacionados ao uso de métodos contraceptivos e relatam ter deixado de usar os métodos de contracepção. 
Outros importantes dados desse estudo são referentes ao desfecho da gravidez indesejada ou não planejada, onde se colheu dados sobre a reação do parceiro e da própria mulher. Nessa ótica, vislumbrou-se que após a descoberta da gravidez a boa parte dos companheiros aceitaram bem a situação (38\%), mas a maioria das mulheres apontaram que o companheiro não achava que era o momento (33\%) ou não queria ter filhos (13\%). Como resultado da pesquisa, apresenta-se ainda que ao descobrir a gravidez a maior parte das mulheres , não pensaram em interrompe-la (74\%), destacando-se entre os motivos a vontade pessoal (28\% ) em ter um filho. Como dado negativo da apuração dessas variáveis tem-se o fato de que entre as mulheres que pensaram em interromper a gravidez (26\%), metade delas apontaram como razão a falta de condições financeiras (13\%) para criar um filho. As demais informações podem ser observadas na Tabela 3.

Tabela 3. Desfecho da atual gravidez apresentado pelas participantes do estudo. Vitória da Conquista e Itambé, Bahia, Brasil, 2016.

\begin{tabular}{lll}
\hline Variáveis & $\mathrm{N}$ & $\%$ \\
\hline Reação do Companheiro & 38 & $38 \%$ \\
Queria a gravidez & 33 & $33 \%$ \\
Queria esperar, não era o momento & 16 & $16 \%$ \\
Não queria ter filhos & 13 & $13 \%$ \\
Foi indiferente ao acontecido & & \\
& & $26 \%$ \\
Pensou em Interromper a Gravidez & 26 & $13 \%$ \\
Sim & 13 & $07 \%$ \\
Falta de Condições Financeiras & 07 & $03 \%$ \\
Não queria o filho & 03 & $02 \%$ \\
Não queria filhos no momento & 02 & $01 \%$ \\
Não vivia com o companheiro & 01 & $74 \%$ \\
Queria trabalharlestudar & & $07 \%$ \\
Não & 74 & $14 \%$ \\
Medo de complicações de saúde & 07 & $04 \%$ \\
Motivo religioso & 14 & $01 \%$ \\
Reação positiva do companheiro & 04 & $09 \%$ \\
Dispunha de condições financeiras & 01 & $28 \%$ \\
Apoio da família & 09 & $11 \%$ \\
Vontade Pessoal & 28 & $\mathbf{1 0 0 \%}$ \\
Outro & 11 & $\mathbf{1 0 0}$ \\
TOTAL & & \\
\hline
\end{tabular}

Mediante os dados apresentados, observa-se que a maioria das gestantes apresentou uma boa aceitação frente à descoberta de uma gravidez não planejada, fato que congrega a visão da maioria dos companheiros, pois muitos tiveram uma boa aceitação da gestação. Sanches (2013), 
Id on Line Revista Multidisciplinar e de Psicologia

Id on Line Multidisciplinary and Psycology Journal

também evidenciou tal resultado em seu estudo, pois ao questionar sobre a reação no momento da descoberta da gravidez, a maioria das mulheres relatou que os parceiros tiveram uma boa aceitação, mas relataram enfrentar certa dificuldade no começo, por se tratar de um fator do qual decorreram várias mudanças na sua vida.

Por essa situação, muitas vezes as mulheres encaram a gravidez não planejada como um período de grandes conflitos e dificuldades e muitas pensam em interromper a gravidez. Nesse estudo um número pequeno de mulheres assinalaram esse desejo, mas como salienta Sanches (2013), uma gravidez não planejada pode gerar sentimentos distintos, provocando emoções como alegria e bem estar ou culpa, frustração e vergonha.

Sobre essa questão, Maldonado (2002 apud SANCHES, 2013) enfatiza que a gravidez não planejada, pode gerar atitudes inicialmente de desacordo e posteriormente de aceitação, após toda a problemática, com a vivência e apoio de familiares, companheiros e possíveis realizações de sonhos futuros, onde a vida vai se organizando e a gestação foi sendo mais bem aceita e esperada. Coelho et al (2012), destaca que no Brasil, o grande número de gravidezes não planejadas tem como desfecho o aborto, constituindo fator importante de morbidade e mortalidade materna. Essas mortes atingem, sobretudo, mulheres jovens e pobres com maior dificuldade de acesso a serviços de saúde por questões sociais e econômicas

Outra abordagem do estudo é apresentada na Tabela 4 e se refere ao uso de métodos contraceptivos por parte das mulheres grávidas. Os dados coletados mostram que a grande parte das mulheres já tentaram evitar a gravidez (85\%). Entre os métodos contraceptivos mais usados destacam-se as injeções (34\%), as pílulas (30\%) e a camisinha (18\%). Ao se averiguar sobre qual o último método contraceptivo utilizado no mês da gravidez, nota-se que as pílulas (30\%) passam a ser as mais utilizadas, seguidas das injeções $(21 \%)$ e da camisinha (24\%), mostrando que muitas mulheres trocaram de método, sendo que essa troca na grande maioria das vezes foi escolha da própria mulher $(62 \%)$ por vários motivos entre eles reações indesejadas e indisponibilidade do método na unidade.

Tabela 4. Uso de métodos contraceptivos apresentado pelas participantes do estudo. Vitória da Conquista e Itambé, Bahia, Brasil, 2016.

\begin{tabular}{lll}
\hline Variáveis & $\mathrm{N}$ & $\%$ \\
\hline Já tentou evitar a gravidez? & & \\
Sim & 85 & $85 \%$ \\
Não & 15 & $15 \%$ \\
\hline
\end{tabular}


Id on Line Revista Multidisciplinar e de Psicologia

\section{Método contraceptivo mais usado}

Nunca usou $\quad 15 \%$

$\begin{array}{lll}\text { Pílulas } & 30 & 30 \%\end{array}$

$\begin{array}{lll}\text { Injeções } & 34 & 34 \%\end{array}$

DIU - Dispositivo Intrauterino $\quad 02 \quad 02 \%$

Tabela/Método do Muco $\quad 01 \quad 01 \%$

$\begin{array}{lll}\text { Camisinha } & 18 \%\end{array}$

$\begin{array}{lcr}\text { Método contraceptivo mais usado após troca } & & \\ \text { Não estava usando } & 18 & 18 \% \\ \text { Pílulas } & 30 & 30 \% \\ \text { Injeções } & 21 & 21 \% \\ \text { Tabela/Método do Muco } & 06 & 06 \% \\ \text { Camisinha } & 24 & 24 \% \\ \text { Coito Interrompido } & 01 & 01 \%\end{array}$

\section{Quem decidiu pela troca}

$\begin{array}{lll}\text { Você } & 62 & 62 \%\end{array}$

$\begin{array}{lll}\text { Parceiro } & 13 & 13 \%\end{array}$

$\begin{array}{lll}\text { Os dois } & 25 & 25 \%\end{array}$

Total

100

$100 \%$

Em consonância com esses resultados, Sanches (2013) em seu estudo, aponta um resultado semelhante quanto a abordagem sobre o uso de métodos contraceptivos, pois a maioria das mulheres participantes da pesquisa indicaram estar fazendo uso de algum método contraceptivo quando descoberta a gravidez não planejada. Percebe-se assim, que o uso de métodos não necessariamente ocorre de forma contínua e consistente, gerando situações de vulnerabilidade contraceptiva.

Quanto a tipologia de método contraceptivo utilizado, os dados de Ciantelli et al (2012), corroboram com esse trabalho ao passo que o mesmo relata que $64,3 \%$ das gestantes envolvidas em seu estudo engravidaram referindo utilizar anticoncepcional oral regularmente, 21,4\% afirmavam utilizar somente preservativos, sendo que apenas 14,3\% não faziam uso de método algum. Esses dados estão bem próximos dos evidenciados nesse trabalho, mostrando que existem sérios problemas quanto ao processo de acompanhamento das mulheres na utilização dos métodos contraceptivos.

No entanto, o dado verificado que mais chama atenção nesse estudo refere-se a troca de método contraceptivo, pois evidenciou-se que a maioria das mulheres citam ter engravidado após a mudança do método contraceptivo, ou seja, alegam que o método que estavam utilizando no mês de sua gravidez "falhou" ou durante a troca do método engravidou. Sobre essa questão 
Id on Line Revista Multidisciplinar e de Psicologia

Id on Line Multidisciplinary and Psycology Journal

Dias e Spinola (2007 apud CIANTELLI, 2012), enumeram que as mulheres não conhecem os métodos e trocam constantemente, sem se preocupar com o tempo de efeito da contracepção, deixando de respeitar os prazos para a devida ação.

Numa análise mais direcionada dos resultados, percebe-se que as pílulas, camisinhas e injeções foram os métodos mais usados no mês que a mulher engravidou. No entanto, esses métodos são comprovadamente seguros para evitar a gravidez, sendo, portanto a sua errônea utilização os prováveis promotores das gravidezes verificadas.

Esse fato leva a percepção de que as mulheres não devem estar utilizando corretamente os métodos contraceptivos. O estudo de Paz e Ditterich (2009) confirma essa evidenciação, pois nos discursos por eles coletados, percebe-se que as mulheres grávidas participantes da pesquisa confundiram as reações adversas com as indicações e contraindicações do método contraceptivo em uso, então assim é possível perceber que as mulheres sabem que existem os métodos, porém desconhecem sua ação e tempo de eficácia.

Afirma-se assim, a necessidade de que a anticoncepção deva ser orientada segundo as características de cada método, para que a mulher escolha o contraceptivo que melhor se adeque ao seu estilo de vida,

Por fim, esse estudo buscou informações sobre a participação das mulheres no planejamento familiar e sobre as informações prestadas nas instituições de saúde pelos profissionais responsáveis (TABELA 5). Nesse contexto, a maior parte das mulheres aponta nunca ter participado de encontros do Planejamento Familiar (67\%), não ter recebido visitas da equipe de Saúde da Família (93\%) e não terem sido instruídas sobre o planejamento da gravidez (75\%) pela instituição de saúde. Em relação a avaliação do enfermeiro no planejamento familiar, das 33 mulheres da amostra que apontaram já ter participado de encontros, somente 19\% relataram ter recebido informações sobre o planejamento da gravidez e a importância do devido acompanhamento da mesma.

Tabela 5. Dados relativos à participação no Planejamento Familiar e informações prestadas pelas instituições de saúde, apresentados pelas participantes do estudo. Vitória da Conquista, Bahia, Brasil, 2016.

\begin{tabular}{lll}
\hline Variáveis & N & $\%$ \\
\hline Já participou de encontros do Planejamento Familiar? & & \\
Nunca participei de encontros & 67 & $67 \%$ \\
Já participei & 33 & $33 \%$ \\
& & \\
\hline
\end{tabular}


Id on Line Revista Multidisciplinar e de Psicologia

Id on Line Multidisciplinary and Psycology Journal

\section{Avaliação do Enfermeiro no Planejamento Familiar}

Não foi atendida por um Enfermeiro na Unidade de Saúde

Foi atendida pelo profissional, mais não se falou sobre planejamento da gravidez.

Foi atendida pelo profissional que fez orientações sobre planejamento da gravidez e importância de acompanhamento

\section{Visita da equipe de Saúde da Família}

Já recebi, mas não foi abordado o tema gravidez ou planejamento $\quad 07 \quad 07 \%$ familiar

Não recebi

\section{Recebeu informações da Unidade de Saúde da Família?}

Sim, recebi importantes informações que me ajudaram a preparar para a gravidez

Não, o(a) profissional que me atendeu não passou informações relevantes

Não fui instruída

Mediante tais resultados, percebe-se que a ampla maioria das mulheres participantes desse estudo não participaram de encontros do Planejamento Familiar ou receberam visitas da equipe de Saúde da Família. Esses dados apontam certa deficiência no processo de instrução e acompanhamento das mulheres pelas unidades básicas de saúde.

Sobre essa situação, Sanches (2013) apresenta que o Planejamento Familiar não consegue atingir a maioria das mulheres devido a falta de participação das mesmas nos encontros nas Unidades de Saúde. As justificativas são as mais variadas, sendo relatado: falta de tempo para comparecer, devido ao trabalho ou outros compromissos, ou mesmos a falta de um convite por parte da equipe de saúde da família, fato também verificado nesse estudo. Nessa mesma ótica, Evangelista, Barbieri e Silva (2015) apontam que seu estudo, a não participação no programa de Planejamento Familiar está ligado ao desconhecimento, seguido de falta de interesse, sendo esses os principais motivos alegados resultado esse que corrobora com o evidenciado nesse estudo.

Quando ocorre o encontro, outro problema se verifica, pois não são prestadas as devidas informações sobre o planejamento. Comprovando tal situação, Coelho et al (2012), aponta que, em estudos realizados no sistema público de saúde, a variedade de métodos anticoncepcionais é limitada, com provisão irregular e distanciamento de outras demandas das mulheres. Isso demonstra a necessidade da equipe de saúde realizar atividades preventivas continuas, para oferecer a clientela os conhecimentos necessários a respeito dos métodos contraceptivos. 
Id on Line Revista Multidisciplinar e de Psicologia

Id on Line Multidisciplinary and Psycology Journal

Nessa mesma ótica Silveira (2012), enumera que as palestras realizadas, são desmotivantes e na grande parte não é tomada nenhuma iniciativa pelos órgãos competentes a respeito disto, existindo uma grande dificuldade na comunicação, sendo esse um ponto crucial, pois os enfermeiros devem atuar capacitando os agentes comunitários para eles possam desenvolver um trabalho significativo nas comunidades.

Percebe-se assim, uma enorme dificuldade no atendimento a população feminina, pois necessita-se aprofundar o conhecimento das mulheres sobre os métodos contraceptivos, e esse aprofundamento está atrelado ao potencial instrucional das unidades de saúde, que não conseguem realizar atividades preventivas continuas para essa clientela.

Isso se evidencia, pois mesmo com o alto número de mulheres usando métodos contraceptivos, ainda não conseguiu se reduzir o número de gestações não planejadas. Prietsch et al (2011) aponta que este aspecto precisa ser considerado para se reavaliar programas de planejamento familiar e as visitas das equipes de saúde da família, visando potencializar as informações sobre o planejamento da gravidez e uso de contraceptivos.

Destaca-se assim, a importância da atuação do Enfermeiro no acompanhamento à saúde sexual da mulher, buscando realizar um trabalho voltado para uma abordagem integral, intervindo na desinformação e na dificuldade de acesso à contracepção, através da captação das mulheres no programa de planejamento familiar.

\section{Considerações Finais}

Mediante as conceptualizações e resultados obtidos com esse estudo, percebeu-se que a gravidez não planejada em mulheres atendidas no pré-natal da unidades de saúde, são muitas vezes resultado do uso inadequado dos métodos contraceptivos, fato esse que pode ser relacionado com alguns aspectos socioeconômicos da população pesquisada, bem como pela baixa participação no planejamento familiar e nas ações das equipes de saúde da família.

Assim, evidencia-se a importância da atuação do enfermeiro no tocante a prevenção, orientação e controle, bem como das equipes de saúde da família, como principais interlocutores do planejamento familiar. 
Id on Line Revista Multidisciplinar e de Psicologia

Id on Line Multidisciplinary and Psycology Journal

Esse estudo também permitiu verificar os possíveis desfechos que a gravidez não planejada poderia causar ter a partir da sua descoberta. Foi evidenciado que a gravidez não planejada está diretamente relacionada a algumas situações socioeconômicas, à vida familiar e conjugal, aos aspectos biopsicossociais. Assim, os dados apontam que algumas mulheres deste estudo, enfrentaram as dificuldades impostas por esta gravidez não planejada, principalmente devido a aceitação da família e dos companheiros, pois essa situação influenciaria diretamente na vida cotidiana pessoal e profissional.

O trabalho permitiu vislumbrar que a grande maioria das mulheres tem acesso aos métodos contraceptivos disponibilizados nas unidades de saúde, destacando-se as pílulas, as injeções e a camisinha. No entanto, mesmo de posse desses anticonceptivos, e utilizando-os cotidianamente, as mulheres relataram ter engravidado.

Esse fato é resultado direto da falta de conhecimento e devida utilização dos métodos contraceptivos, que possuem especificidades quanto a dosagem, tempo de ação, temo de duração da contracepção, dentre outros conhecimentos, normalmente desconhecidos por parte da população feminina, que em sua maioria ainda não consegue colocar em prática a informação para evitar a gestação.

Com base nos resultados e discussões propostas nesse estudo, pode-se enumerar a necessidade de se garantir o acesso aos métodos contraceptivos com a devida orientação, sendo indispensável para isso a ampliação dos espaços de diálogo, discussão e orientação sobre o planejamento familiar, com atuação destacada do enfermeiro e das equipes de saúde da família, no tocante ao desenvolvimento de ações educativas, preventivas e de promoção da saúde junto à essa população.

Por fim, considera-se aqui a importância da realização de outros estudos que investiguem mais profundamente outras demandas que envolvam as gravidezes não planejadas, principalmente no que tange ao acompanhamento das gestantes, e a devida utilização dos métodos contraceptivos. 
Id on Line Revista Multidisciplinar e de Psicologia

Id on Line Multidisciplinary and Psycology Journal

\section{Referências}

BAYER. Descuido e uso incorreto de métodos contraceptivos são a principal causa da gravidez não planejada. Publicado em 2014. Disponível em <https://www.bayer.com.br/midia/noticias/visualiza-noticia.php?codNoticia=26-de-setembrodia-mundial-da-prevencao-da-gravidez-nao-planejada>. Acesso em 10 de maio de 2016.

BERQUÓ E.; LIMA L. P. Planejamento da fecundidade: Gravidezes não desejadas - PNDS 1996 e 2006. Cap 7 pag 135-149 In: Brasil. Ministério da Saúde. Pesquisa Nacional de Demografia e Saúde da Criança e da Mulher (PNDS) 2006: dimensões do processo reprodutivo e da saúde da criança/Ministério da Saúde, Centro Brasileiro de Análise e Planejamento. Brasília: Ministério da Saúde, 2009.

BRITO, C. N. O.. Gravidez não pretendida e sua associação com depressão pós-parto entre mulheres atendidas no pré-natal pela estratégia de saúde da família no Recife-PE. Dissertação (mestrado) - UFPB, CCS, Programa de Pós-Graduação em Saúde Coletiva, 2013.

CIANTELLI, G. L. A gravidez não planejada em um bairro periférico de Sorocaba-SP. Revista Fac. Ciênc. Méd. Sorocaba. 2012.

COELHO, E. A. C. Associação entre gravidez não planejada e o contexto socioeconômico de mulheres em área da Estratégia Saúde da Família. Acta Paul Enferm. 2012.

EVANGELISTA, C. B.; BARBIERI, M.; SILVA, P. L. N. J. Gravidez não planejada e fatores associados à participação em programa de planejamento familiar. Revista de Pesquisa e Cuidado Fundamental Online. abr./jun. 7(2), 2015.

FINER, L. B.; ZOLNA, M. R. Unintended pregnancy in the United States: incidence and disparities, 2006. Contraception, v.84, n.5, 2011.

FINOTTI, Marta. As implicações da gravidez não planejada de adolescentes no Brasil. Publicado em 2015. Disponível em <http://www.febrasgo.org.br/site/?p=11633>. Acesso em 10 de maio de 2016.

IBGE. Infográficos: dados gerais do município. Disponível em: <http://cidades .ibge.gov.br/xtras/perfil.php?codmun=270630>. Acesso em 19 de março de 2016.

NERY, I. S. Fatores associados à reincidência de gravidez após gestação na adolescência no Piauí, Brasil. Revista Epidemiol. Serv. Saúde, Brasília, 2015.

PAZ, E. C. M.; DITTERICH, R. G. O conhecimento das mulheres sobre os métodos contraceptivos no planejamento familiar. Rev. Gestão \& Saúde, Curitiba, 2009.

PRIETSCH, S. O. M. et al. Gravidez não planejada no extremo Sul do Brasil: prevalência e fatores associados. Caderno Saúde Pública, Rio de Janeiro, 2011. 
SARDINHA, A. S, et al. Levantamento das causas da gravidez em mulheres atendidas na unidade básica de saúde São João Evangelista, Paracatu-MG. Revista de Medicina da Faculdade de Atenas de Paracatu - MG. Paracatu - MG, 2014.

SANCHES, N. C. Gravidez não planejada: a experiência das gestantes de um município do interior do Estado de São Paulo. Dissertação de Mestrado, Escola de Enfermagem de Ribeirão Preto/USP. Ribeirão Preto-SP: USP, 2013.

SILVEIRA, R. R. Atuação do enfermeiro do programa saúde da família na prevenção e controle da gravidez precoce. (Monografia). Universidade Salgado de Oliveira, São GonçaloRJ: 2012.

Como citar este artigo (Formato ABNT):

RODRIGUES, C.D.S. A gravidez não planejada de mulheres atendidas no pré-natal das Unidades Básicas de Saúde . Id on Line Revista Multidisciplinar e de Psicologia, Nov-Dez. de 2016, vol.10, n.32, p. 70-87. ISSN: 1981-1179.

Recebido: 05/11/2016

Aceito: 08/11/2016 